\title{
A phase 2, single-arm trial of neoadjuvant axitinib plus avelumab in patients with localized renal cell carcinoma (RCC) who are at high risk of relapse after nephrectomy (NEOAVAX)
}

Keywords: renal cancer, avelumab, axitinib, phase 2, neoadjuvant

Word count: 2466

Abstract: Surgery is the standard treatment for non-metastatic RCC. Despite curative intent, patients with a high risk of relapse have a 5-year metastasis-free survival rate of only $30 \%$ and prevention of recurrence is an unmet need. In a phase 3 trial (JAVELIN Renal 101), progression free survival (PFS) of axitinib + avelumab was superior to sunitinib with a favourable objective response rate (ORR) and no added toxicity profiles as known for axitinib or avelumab single agent . NEOAVAX is designed as open label, single arm, phase II trial with a Simon's two-stage design evaluating neoadjuvant axitinib + avelumab followed by complete surgical resection in 40 patients with high-risk non-metastatic clear-cell (cc) RCC. Primary endpoint is remission of the primary tumour (RECIST 1.1) following neoadjuvant therapy. Secondary endpoints include DFS, OS, rate of metastasis and local recurrence, safety, and tolerability. Exploratory endpoints include investigation of effects on neo-angiogenesis, immune infiltrates and MDSC components to support a rationale for the combined use of axitinib and avelumab. (NCT03341845).

\section{Introduction:}

Renal cell carcinoma (RCC) accounts for $2.4 \%$ of adult malignancies and constitutes $95 \%$ of renal tumours [1]. Globally, some 403,262 new cases were reported for 2018 with 175,098 deaths from this disease [2]. Surgical complete resection is currently the only curative treatment for RCC, including patients with locally advanced RCC or limited metastatic disease. However, 
these patients carry a high risk to develop locally recurrent disease and distant metastases. According to the validated Leibovich and University of California Los Angeles Integrated Staging System (UISS) risk scores high-risk patients have a 2-year local or systemic failure rate of 57.5 $\%$ and a 3-year metastasis-free survival of only $37.1 \%[3]$. High-risk patients with no evidence of disease following complete resection may therefore benefit from adjuvant and neo-adjuvant systemic treatment strategies which primarily aim to prolong disease-free (DFS) and ultimately overall survival (OS).

Vascular endothelial growth factor receptor (VEGFR)-targeted therapy has improved progression-free survival (PFS) and OS in metastatic RCC[4]. Based on the efficacy in advanced disease, several randomized phase III trials investigated adjuvant therapy targeting VEGFR after resection of high-risk non-metastatic RCC. To date, the results of several trials with multi-tyrosine kinase inhibitors (TKI) as sunitinib, pazopanib, sorafenib and axitinib, have been published. [5] [6]. The results did not show an OS benefit with inconsistent DFS benefit. A meta-analysis of these studies clearly revealed a poor benefit-to-harm ratio and guidelines do not recommend adjuvant VEGFR-targeted therapy in this group of patients [7].

Nivolumab, a monoclonal antibody targeting and blocking the inhibitory Programmed Death-1 (PD-1) receptor was first introduced into the treatment paradigm of metastatic RCC when a phase 3 trial showed superior OS with nivolumab compared to everolimus after 1 or 2 lines of previous VEGFR-targeted therapy[8]. Immune checkpoint inhibitors have since then been investigated in several phase 3 trials as combination of ipilimumab and nivolumab, or in combination with VEGF-targeted therapy in patients with previously untreated metastatic RCC, all against the current standard of care sunitinib [9]. The combination of nivolumab and ipilimumab has revealed superior OS compared to sunitinib, as first-line treatment of $c c R C C$, in addition, the ORR of nivolumab and ipilimumab was $41.6 \%$ compared to $26.5 \%$ for sunitinib ( $p$ 
$<0.0001$ ) and in a 30 -months update $11 \%$ of patients receiving combination therapy achieved a complete response (CR) compared to $1.2 \%$ of patients on sunitinib $[9,10]$.

Based on the rationale that immunotherapy is more effective in eliminating circulating tumour cells and micrometastases than VEGFR-targeted therapy, four randomized controlled phase III trials are planned to test adjuvant immune checkpoint inhibitors in patients with non-metastatic RCC and high risk of recurrence. [11, 12].

However, preclinical and early clinical research suggests that there may be significantly greater therapeutic efficacy of neoadjuvant immune therapies compared to an adjuvant approach to eradicate distant metastases in combination with primary tumour resection [13]. Since immunotherapy importantly depends on antigen-recognition via the T cell receptor (TCR), it is hypothesized that T cell checkpoint inhibition is of greatest value at the moment of TCR triggering, which is when the tumor is still in situ, therefore in the neoadjuvant and not adjuvant setting. This hypothesis, when supported by further clinical evidence, may have significant consequences for the use of checkpoint targeting antibodies as adjuvant therapies. An effective preoperative treatment may be superior in preventing disease recurrence or metastasis and ultimately impact on survival. This has resulted in several neoadjuvant phase $1 / / l$ studies which are currently ongoing. A phase I neoadjuvant trial in patients with RCC has investigated the lymphocytic infiltration, safety and early efficacy of pembrolizumab [14]. In addition, two safety and efficacy studies investigate neoadjuvant nivolumab, one in the non-metastatic setting and one as pre- and post-operative therapy in metastatic RCC (ADAPTeR) $[15,16]$. Another neoadjuvant phase $1 \mathrm{~b}$ study tests safety and efficacy of durvalumab plus tremelimumab in advanced RCC [17]. In metastatic RCC, tumour shrinkage is a prognosticator for OS when treated systemically [18]. A larger data set showed that the degree of tumour shrinkage correlated with the prognosis in mRCC, suggesting that deep responses are a desirable treatment goal [19]. Patients with tumour shrinkage of at least $60 \%$ achieved a median OS of 55 months, supporting this notion. 
As responses may have a late onset, an early marker was sought as a prognosticator. $10 \%$ tumour shrinkage at 6 weeks of medical treatment correlated with improved OS, suggesting that tumour shrinkage is a prognostic factor which occurs early during the course of medical therapy in RCC $[20,21]$. Overall, the amount of tumour shrinkage and its early onset are prognostic markers in $\mathrm{mRCC}$; however, it remains unclear whether these findings can be replicated in locally advanced disease.

Neoadjuvant studies provide a unique opportunity to investigate mechanisms of response and resistance to immune checkpoint inhibition and to identify biomarkers predicting these outcomes. Recent research on intratumoural immune components such as tumour infiltrating lymphocytes (TIL) or myeloid derived suppressor cells (MDSC) after pretreatment of human renal cell carcinoma suggest a potential synergism for TKI with anti-PD-(L)1 therapy that could be exploited [22]. In terms of downsizing tumours by pretreatment, axitinib appears to be more effective than sunitinib when comparing single-arm trials that have been performed with each drug [23]. However, the immunomodulatory effect of axitinib has been poorly defined. Since axitinib and anti-PD-(L)1 therapy would make for a potential synergism, two phase lb dosefinding studies to evaluate safety, pharmacokinetics and pharmacodynamics of avelumab, an anti-PD-L1 monoclonal antibody, or pembrolizumab, an anti-PD1 monoclonal antibody, in combination with axitinib were performed in metastatic RCC [24, 25]. The combinations of avelumab and axitinib or pembrolizumab and axitinib led to an observed ORR of $58.2 \%$ and 73\% respectively and toxicity profiles typical for VEGFR-treatment and anti-PD-(L)1 therapy. Both combinations have been tested in phase III trials against sunitinib in untreated metastatic $\mathrm{RCC}[26,27]$. While the combination of avelumab and axitinib demonstrated superior outcome for PFS and updated OS data are pending [26], the combination of pembrolizumab and axitinib improved PFS and OS at 12 months compared to sunitinib with a hazard ratio (HR) of 0.53 (95\% Confidence Interval (Cl) 0.38-0.74) [27]. 


\section{NEOAVAX trial}

We describe the design of a single-arm, open-label phase 2 trial of neoadjuvant avelumab and axitinib in patients with intermediate to high-risk non-metastatic clear-cell RCC (NCT03341845).

\section{Objectives}

The primary objective of this trial is to evaluate efficacy and safety of neoadjuvant axitinib and avelumab in patients with localized renal cell carcinoma with intermediate to high risk of recurrence.

\section{Key eligibility criteria}

Key inclusion criteria are: planned radical or partial nephrectomy with curative intent; biopsy proven ccRCC; clinical high risk defined as cT1b-cT2a grade (G) 4, cT2b G3, cT3a G3-4, cT3bcT4 any G cNOM0, or cT any cN1M0; World Health Organization (WHO) performance status of $0-1$, and completely resectable primary tumours. Key exclusion criteria are: metastatic disease, corticosteroid or immunosuppressive systemic treatment, active autoimmune disease, prior systemic treatment for RCC including immunotherapy, biologic therapy, investigational therapy or hormonal therapy. Other eligibility criteria are adequate coagulation, hematological, hepatic and renal function, age $\geq 18$ years and signed informed consent.

\section{Study design}


NEOAVAX is a single-arm, open-label phase 2 trial of neoadjuvant avelumab and axitinib followed by complete surgical resection in patients with intermediate to high-risk locally advanced but non-metastatic clear-cell RCC (figure 1). The trial has appropriate institutional review board approval and informed consent is obtained from the participants involved. For the purpose of this study, the definition of intermediate to high risk will be based on clinical TNM and biopsy nuclear grade since the full pathological specimen is not available. Patients receive axitinib $5 \mathrm{mg} \mathrm{BID}$, which is increased to $7 \mathrm{mg}$ BID after 4 weeks and a maximum of $10 \mathrm{mg}$ BID after 8 weeks if tolerated, in combination with avelumab $10 \mathrm{mg} / \mathrm{kg}$ iv Q2W for 6 cycles. Patients with an increase of the tumour from baseline at CT after the first 6 weeks or no change after dose escalation will be taken off-trial and undergo surgery. All others will complete 3 months of study treatment followed by resection. The primary endpoint of the trial is remission of the primary tumour (RECIST 1.1) following neoadjuvant avelumab and axitinib (Box 1). Secondary endpoints are disease-free survival (DFS), OS, rate of metastasis and local recurrence, safety, and tolerability. Exploratory endpoints include investigation of effects on neo-angiogenesis, immune infiltrates and MDSC components to support a rationale for the combined use of axitinib and avelumab.

Figure 1: Study design of NEOAVAX 


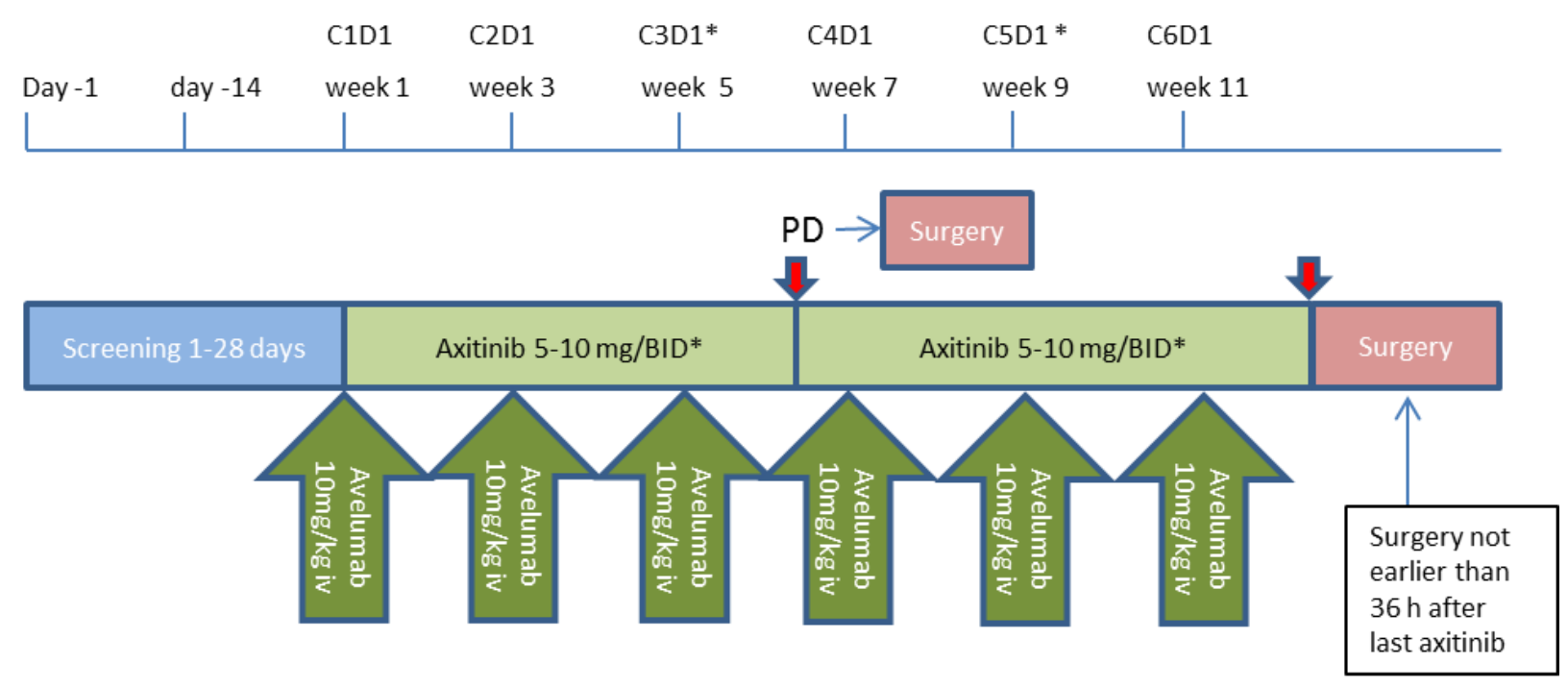

*after week 4: if tolerated well, switch to $7 \mathrm{mg}$ Axitinib BID

after week 8: if tolerated well, switch to $10 \mathrm{mg}$ Axitinib BID

\section{Box 1: NEOAVAX study endpoints}

\section{Primary end points}

- Remission of the primary tumour following neoadjuvant axitinib and avelumab according to RECIST 1.1

\section{Secondary end points}

- Safety and adverse events of the combination of axitinib with avelumab

- Pathological response (no response, >50\% tumour reduction, >90\% tumour reduction, and $\mathrm{CR}$ ) as described in [9]

- DFS, OS, rate of metastasis and local recurrence

- Clavien Dindo classification of surgical morbidity

\section{Exploratory endpoints}

- Collection of peripheral blood and tissue (both fresh frozen and paraffin embedded) on pretreatment biopsies and post-treatment partial/total nephrectomies for:

- immune infiltrate and MDSC components.

- concomitant effects on neoangiogenesis

- genomic profiling and sequencing.

DFS disease free survival; OS overall survival; RECIST: Response Evaluation Criteria In Solid Tumours; MDSC myeloid derived suppressor cells;

\section{Evaluations}

Response and DFS are evaluated per RECIST 1.1 based on investigator review. Response assessments include a baseline documentation of overall tumour burden and target lesions at entry and after 6 weeks and 3 months of pretreatment. Therapeutic drug monitoring for axitinib 
will be performed after the first two weeks of standard $5 \mathrm{mg}$ BID and after doses will be increased to $7 \mathrm{mg}$ BID if tolerated. Therapeutic drug monitoring will be repeated at 4 weeks and if necessary and tolerated doses will increase to $10 \mathrm{mg} \mathrm{BID.} \mathrm{Patients} \mathrm{will} \mathrm{undergo} \mathrm{a} \mathrm{CT} \mathrm{scan} 6$ weeks after initiating therapy. Those with an increase of the tumour after the first 6 weeks or no change after dose escalation will be taken off trial and undergo surgery. All others will continue for a maximum of 3 months followed by a CT scan. After intervention patients will be followed by CT scan of the abdomen and thorax every 3 months in the first year, every 6 months in the second year and once a year in year 3 to 10, or at progression/recurrence.

The intensity of an adverse event will be graded throughout the trial according to the National Cancer Institute Common Toxicity Criteria (NCI CTC) v 4.03. Adverse events that cannot be graded using the NCI CTC will be graded as mild (asymptomatic), moderate (symptomatic but not interfering significantly with function) or severe (causing significant interference with function). Given the potential risk for delayed immune-related toxicities, safety follow-up must be performed up to 90 days after the last dose of avelumab administration.

Exploratory assessments will include full characterization of the immunological characteristics of the tumour microenvironment, including angiogenesis and hypoxia at the protein and gene expression level using nanostring sequencing and an expression panel, including the Tumour Inflammation Signature (TIS) which has been shown to predict response to immune checkpoint blockade therapy across multiple solid tumours. RNA will be extracted from pre- and posttreatment fresh frozen tissue to assess changes . In addition, whole exome sequencing and germline DNA comparison will be performed on DNA extracted from pre- and post-treatment fresh frozen tissue samples and blood samples, to determine tumour mutational burden in pretreatment samples and assessment of genetic changes that might lead to treatment resistance in post-treatment samples. Finally, clonality of the T-cell population will be determined by deep sequencing of TCR V $\beta$. 


\section{Statistical analysis and sample size estimation}

Presurgical treatment of tumours results in downsizing by $10-11 \%$ of the maximal diameter, but partial remissions are rare. However, a previous axitinib phase II neoadjuvant trial in 24 locally advanced tumours has demonstrated a median primary tumour diameter reduction of 28.3 $\%[23]$. This study is therefore designed to detect a $25 \%$ remission rate of the primary tumour with a one-sided alpha of 0.05 and a power of $80 \%$ using a two-stage minimax design developed by Simon [28], with a null hypothesis of $10 \%$ remission rate.

This design allows for early termination of the study if the drug is associated with low activity. First, 22 patients will be treated and if at most 2 remissions according to RECIST 1.1 are observed in the primary tumour, the study will be stopped for insufficient activity on the primary tumour. If at least 3 remissions are observed, 18 additional patients will be included to a total of 40 patients. If at least 8 primary tumours of these patients have a remission the treatment will be declared to have sufficient activity on the primary tumour and treatment choice. Otherwise the treatment will be declared not sufficiently active on the primary tumour.

\section{Conclusion and future perspective}

NEOAVAX is a neoadjuvant trial of a combination of an anti-PD-L1 monoclonal antibody, avelumab, and a VEGFR-TKI, axitinib, in patients with high risk of relapse after surgery with curative intent. Enrollment began in June 2018, and the estimated date of primary end point data availability is June 2021. Results from this trial will determine whether the combination of avelumab and axitinib given as neoadjuvant treatment during 3 months will result in a significant remission rate of primary tumours and locoregional lymph node metastases in patients with locally advanced clear-cell RCC. The secondary objectives will provide information on safety of 
neoadjuvant therapy, surgical complications, pathological responses, DFS and OS as well as an opportunity to investigate mechanisms of resistance and response on a molecular level. This is of importance because immune checkpoint inhibitors can lead to complete and durable responses in a minority of patients, especially when combined with other checkpoint inhibitors or VEGF-inhibiting agents such as bevacizumab and axitinib $[9,24,25]$. In view of the costs and to counsel patients predictors of response to treatment will have to be identified beyond the currently unsatisfactory correlation of outcome with PD-L1 expression status. McDermott et al recently analysed tumour tissue from a phase 2 trial of atezolizumab plus bevacizumab versus sunitinib in metastatic RCC and found that tumour mutation and neoantigen burden were not associated with PFS [29]. Instead, gene expression signatures of angiogenesis, T-effector/IFN-Y response, and myeloid inflammatory signatures were found to be strongly associated with PFS and discriminating between treatment arms. These molecular profiles suggest prediction of outcomes with immune checkpoint inhibition and VEGF targeted therapy. Sequencing of tissue and tumour microenvironment from material obtained from NEOAVAX and other trials in which tumour tissue is pretreated have the potential to offer mechanistic insights into how combination therapy may overcome resistance to immune checkpoint blockade. If results from NEOAVAX, in particular the exploratory analysis of the TIS together with primary tumour shrinkage, support the neoadjuvant use of avelumab and axitinib we will propose a randomized phase 3 trial of avelumab and axitinib against a future standard.

Executive summary

\section{Background}

- patients with locally advanced or high grade RCC are at high risk of relapse

- Currently no adjuvant or neoadjuvant treatment is established

- Combination therapy with immune checkpoint inhibition and VEGF targeted therapy yields superior outcome in metastatic RCC when compared to VEGF targeted therapy alone 
- Mechanisms of response and resistance are not understood

- Neoadjuvant trials may improve outcome by priming the immune system and provide opportunities for translational research of mechanisms of resistance

\section{NEOAVAX trial}

- open label, single arm, phase II trial of neoadjuvant axitinib + avelumab followed by complete surgical resection

- Sample size includes 40 patients with high-risk non-metastatic clear-cell RCC.

- Primary endpoint is remission of the primary tumour (RECIST 1.1) following neoadjuvant therapy.

- Secondary endpoints include DFS, OS, rate of metastasis and local recurrence, safety, and tolerability.

- Exploratory endpoints include investigation of effects on neo-angiogenesis, immune infiltrates and MDSC components and mechanisms of resistance to therapy.

- The trial is registered at clinicaltrials.gov (NCT03341845).

1. Ferlay J, Colombet M, Soerjomataram I et al. Cancer incidence and mortality patterns in Europe: Estimates for 40 countries and 25 major cancers in 2018. European journal of cancer (Oxford, England : 1990) 103 356-387 (2018).

2. Bray F, Ferlay J, Soerjomataram I, Siegel RL, Torre LA, Jemal A. Global cancer statistics 2018: GLOBOCAN estimates of incidence and mortality worldwide for 36 cancers in 185 countries. CA: a cancer journal for clinicians 68(6), 394-424 (2018).

3. Sun M, Shariat SF, Cheng $C$ et al. Prognostic factors and predictive models in renal cell carcinoma: a contemporary review. Eur. Urol. 60(4), 644-661 (2011).

4. Ljungberg B, Bensalah K, Canfield S et al. EAU guidelines on renal cell carcinoma: 2014 update. European urology 67(5), 913-924 (2015).

5. Haas NB, Manola J, Uzzo RG et al. Adjuvant sunitinib or sorafenib for high-risk, non-metastatic renal-cell carcinoma (ECOG-ACRIN E2805): a double-blind, placebo-controlled, randomised, phase 3 trial. Lancet 387(10032), 2008-2016 (2016).

6. Ravaud A, Motzer RJ, Pandha HS et al. Adjuvant Sunitinib in High-Risk Renal-Cell Carcinoma after Nephrectomy. N. Engl. J. Med. doi:10.1056/NEJMoa1611406 (2016). 
7. Bex A, Albiges L, Ljungberg B et al. Updated European Association of Urology Guidelines Regarding Adjuvant Therapy for Renal Cell Carcinoma. Eur. Urol. doi:10.1016/j.eururo.2016.11.034 (2016).

8. Motzer RJ, Escudier B, Mcdermott DF et al. Nivolumab versus Everolimus in Advanced Renal-Cell Carcinoma. N. Engl. J. Med. doi:10.1056/NEJMoa1510665 (2015).

* the pivotal trial introducing nivolumab into the treatment paradigm of metastatic RCC

9. Motzer RJ, Tannir NM, Mcdermott DF et al. Nivolumab plus Ipilimumab versus Sunitinib in Advanced Renal-Cell Carcinoma. The New England journal of medicine 378(14), 1277-1290 (2018).

** based on this trial combination immunotherapy replaced sunitinib as standard in first-line therapy for intermediate and poor risk patients.

10. Tannir NMF, O a; Hammers, H J; Carducci, M a; Mcdermott, D F; Salman, P; Escudier, B;. Thirtymonth follow-up of the phase III CheckMate 214 trial of first-line nivolumab + ipilimumab $(\mathrm{N}+\mathrm{I})$ or sunitinib (S) in patients (pts) with advanced renal cell carcinoma (aRCC). J Clin Oncol 37 (2019).

11. Massari F, Santoni M, Ciccarese C et al. PD-1 blockade therapy in renal cell carcinoma: current studies and future promises. Cancer Treat. Rev. 41(2), 114-121 (2015).

12. Kasenda B, Larkin J, Gore M. Immunotherapies in Early and Advanced Renal Cell Cancer. Prog Tumor Res 42 1-10 (2015).

13. Liu J, Blake SJ, Yong MC et al. Improved Efficacy of Neoadjuvant Compared to Adjuvant Immunotherapy to Eradicate Metastatic Disease. Cancer Discov. 6(12), 1382-1399 (2016).

14. Health UNIO. NCT02212730: A Study Evaluating the Effect of Pembrolizumab (MK-3475) in Participants With Renal Cell Cancer (MK-3475-031). (2014).

15. Nct02575222. Study of Neoadjuvant Nivolumab in Patients With Non-metastatic Stage II-IV Clear Cell Renal Cell Carcinoma. (2016).

16. Nct02446860. A Study of Anti-PD1 (Nivolumab) Therapy as Pre- and Post-operative Therapy in Metastatic Renal Cell Cancer (ADAPTeR) (ADAPTeR). (2016).

17. Nct02762006. Neoadjuvant MEDI 4736 +/- Tremelimumab in Locally Advanced Renal Cell Carcinoma. (2016).

18. Molina AM, Lin X, Korytowsky B et al. Sunitinib objective response in metastatic renal cell carcinoma: analysis of 1059 patients treated on clinical trials. European journal of cancer (Oxford, England : 1990) 50(2), 351-358 (2014).

19. Grunwald V, Mckay RR, Krajewski KM et al. Depth of remission is a prognostic factor for survival in patients with metastatic renal cell carcinoma. European urology 67(5), 952-958 (2015).

20. Krajewski KM, Franchetti Y, Nishino M et al. 10\% Tumor diameter shrinkage on the first followup computed tomography predicts clinical outcome in patients with advanced renal cell carcinoma treated with angiogenesis inhibitors: a follow-up validation study. The oncologist 19(5), 507-514 (2014).

21. Grunwald V, Lin X, Kalanovic D, Simantov R. Early Tumour Shrinkage: A Tool for the Detection of Early Clinical Activity in Metastatic Renal Cell Carcinoma. European urology 70(6), 1006-1015 (2016).

22. Guislain A, Gadiot J, Kaiser A et al. Sunitinib pretreatment improves tumor-infiltrating lymphocyte expansion by reduction in intratumoral content of myeloid-derived suppressor cells in human renal cell carcinoma. Cancer Immunol. Immunother. doi:10.1007/s00262-015-1735-z (2015).

23. Karam JA, Devine CE, Urbauer DL et al. Phase 2 trial of neoadjuvant axitinib in patients with locally advanced nonmetastatic clear cell renal cell carcinoma. European urology 66(5), 874-880 (2014). 
* a phase 2 neoadjuvant trial demonstrating safety and efficacy of axitinib prior to nephrectomy.

24. Atkins MB, Plimack ER, Puzanov I et al. Axitinib in combination with pembrolizumab in patients with advanced renal cell cancer: a non-randomised, open-label, dose-finding, and doseexpansion phase $1 \mathrm{~b}$ trial. The Lancet. Oncology 19(3), 405-415 (2018).

25. Choueiri TK, Larkin J, Oya $\mathrm{M}$ et al. Preliminary results for avelumab plus axitinib as first-line therapy in patients with advanced clear-cell renal-cell carcinoma (JAVELIN Renal 100): an openlabel, dose-finding and dose-expansion, phase 1b trial. The Lancet. Oncology 19(4), 451-460 (2018).

26. Motzer RJ, Penkov K, Haanen J et al. Avelumab plus Axitinib versus Sunitinib for Advanced RenalCell Carcinoma. The New England journal of medicine 0(0), null

** the phase 3 trial demonstrating superior PFS of avelumab and axitinib compared to sunitinib.

27. Rini BI, Plimack ER, Stus V et al. Pembrolizumab plus Axitinib versus Sunitinib for Advanced Renal-Cell Carcinoma. The New England journal of medicine $0(0)$, null

** the phase 3 trial demonstrating superior PFS and OS of pembrolizumab and axitinib compared to sunitinib across all risk groups.

28. Simon R. Optimal two-stage designs for phase II clinical trials. Controlled clinical trials 10(1), 1-10 (1989).

29. Mcdermott DF, Huseni MA, Atkins MB et al. Clinical activity and molecular correlates of response to atezolizumab alone or in combination with bevacizumab versus sunitinib in renal cell carcinoma. Nature medicine 24(6), 749-757 (2018).

* an important trial providing potential predictive biomarkers to distinguish between tumors which are more angiogenesis driven or have an inflammatory microenvironment. 